\title{
Geographic Variation in the Quality and Cost of Care for Patients with Rheumatoid Arthritis
}

\author{
Jason Shafrin, PhD; Arijit Ganguli, MBA, PhD; Yuri Sanchez Gonzalez, PhD; \\ Jin Joo Shim, MS; and Seth A. Seabury, PhD
}

\begin{abstract}
BACKGROUND: There is considerable push to improve value in health care by simultaneously increasing quality while lowering or containing costs. However, for diseases that are best treated with comparatively expensive treatments, such as rheumatoid arthritis (RA), there could be tension between these aims. In this study, we measured geographic variation in quality, access, and cost for patients with RA, a disease with effective but costly specialty treatments.
\end{abstract}

OBJECTIVE: To assess the geographic differences in the quality, access, and cost of care for patients with RA.

METHODS: Using large claims databases covering the period between 2008 and 2014, we measured quality of care metrics by metropolitan statistical areas (MSAs) for patients with RA. Quality measures included use of disease-modifying antirheumatic drugs (DMARDs) and tuberculosis (TB) screening before initiating biologic DMARD therapy. Access to care measures included measured detection and the share of patients with RA who visited a rheumatologist. Regression models were used to control for differences in patient demographics and health status across MSAs.

RESULTS: For the 501,376 patients diagnosed with RA, in the average MSA $64.1 \%$ of RA patients received a DMARD, and $29.6 \%$ of RA patients initiating a biologic DMARD appropriately received a TB screening. Only 17\% (73/430) of MSAs comprised the top 2 Medicare Advantage star ratings for DMARD use. Measured detection was $0.59 \%$ (IQR $=0.47 \%-0.71 \%$; $C V=0.355)$ on average, and $57.6 \%$ (IQR $=48 \%-69 \%$; CV $=0.341)$ of RA patients visited a rheumatologist. MSAs with the highest DMARD use spent \$26,724 (in 2015 U.S. dollars) annually treating patients with RA, $\$ 5,428$ more $(P<0.001)$ than low DMARD-use MSAs, largely because of higher pharmacy cost $(\$ 5,090$ vs. $\$ 7,610, P<0.001)$. However, MSAs with higher DMARD use had lower RA-related inpatient cost ( $\$ 1,890$ vs. $\$ 2,342, P=0.024)$.

CONCLUSIONS: There were significant geographic variations in the quality of care received by patients with RA, although quality was poor in most areas. Fewer than 1 in 5 MSAs could be considered high quality based on patient DMARD use. Access to specialist care may be an issue, since just over half of patients with RA visited a rheumatologist annually. Efforts to incentivize better quality of care holds promise in terms of unlocking value for patients, but for some diseases, this approach may result in higher costs.

J Manag Care Spec Pharm. 2016;22(12):1472-81

Copyright @ 2016, Academy of Managed Care Pharmacy. All rights reserved.

\section{What is already known about this subject}

Two quality measures-use of disease-modifying antirheumatic drugs (DMARDs) and screening for tuberculosis before initiating a biologic DMARD - have been shown to improve health outcomes among patients with rheumatoid arthritis (RA).

For many diseases, health care quality varies significantly by geographic region.

\section{What this study adds}

Patients with RA received low quality of care on average-fewer than 1 in 5 metropolitan statistical areas provide high quality care to patients with RA based on Medicare Advantage's 4- or 5-star standards for DMARD use among patients with RA.

This analysis demonstrated that there is significant variation in measured detection of RA, access to rheumatologists, and quality of care provided across regions.

Regions with high quality of care based on DMARD use have lower RA-related inpatient costs but higher all-cause total costs.

A lthough it is a burdensome and chronic disease, advances in treatments and practice patterns for rheumatoid arthritis (RA) have significantly improved patient health and disease management. Early diagnosis and treatment can mitigate RA's painful and irreversible swelling in the joints, which can spread to a patient's organs if left unmanaged. ${ }^{1}$ Estimates suggest RA is associated with 4 million work loss days, costing employers $\$ 5.8$ billion in direct and indirect costs annually due to sick leave, short- and long-term disability, and worker compensation. ${ }^{2}$

As part of a broader "Triple Aim" movement in the United States to improve health care quality and value in recent years, a number of RA measures have been developed and used by payers to measure quality of care and increase access to care and cost. Organizations such as the American College of Rheumatology and the National Committee for Quality Assurance have developed RA-related quality of care measures and clinical outcomes. ${ }^{3-5}$ These quality measures have been adopted by private and government payers not only to evaluate physician performance, but also to change reimbursement rates. ${ }^{6,7}$ Despite these new quality initiatives, recent research suggests that the quality of care patients with RA currently receive is suboptimal. ${ }^{8,9}$

The purpose of this study was to characterize the care RA patients receive in the United States and how it varies across the country. Specifically, we measured the quality of care, access to care, and cost of care RA patients received. Quality of care was measured based on disease-modifying antirheumatic drug (DMARD) use and conducting a tuberculosis (TB) test before initiating a biologic DMARD. To measure access to care, 
2 additional statistics were used: measured detection of RA by region and share of patients with RA who had visited a rheumatologist in the previous year. Some previous research indicates that quality of care is no better in regions with above average health care expenditures compared with regions with below expenditures and, in some cases, might even be worse. ${ }^{10-13}$ Since future physician reimbursement will be increasingly tied not only to quality but also to resource use (i.e., health care expenditures), we explored whether "high quality" areas were also able to provide the same quality of care with lower health care expenditures.

\section{Methods}

\section{Data Sources}

RA patients were identified from the Truven Health Analytics MarketScan Commercial Claims and Encounters database and Medicare Supplemental and Coordination of Benefits database between July 1, 2008, and June 30, 2014. The MarketScan Commercial database includes medical and pharmacy claims for individuals and their dependents covered by employersponsored private health insurance. The MarketScan Medicare database includes medical and pharmacy claims of Medicareeligible retirees with employer-sponsored supplemental plans. These data were used to measure patient demographics, enrollment, presence of RA and other comorbidities, and resource and expenditure information.

To measure average socioeconomic status in each area, the MarketScan claims data were supplemented with information from the 2013-2014 Area Health Resources File (AHRF). ${ }^{14}$ The AHRF contains county-level information on health facilities, health professionals, measures of resource scarcity, health status, economic activity, and socioeconomic and environment characteristics. Using the AHRF data, we measured the share of minority patients, average household income, share of individuals in poverty, and education levels by county.

\section{Study Population}

We included all U.S. residents in the MarketScan data aged $\geq 18$ years that had $\geq 1$ inpatient or $\geq 2$ outpatient claims with a valid RA diagnosis (International Classification of Diseases, Ninth Revision, Clinical Modification codes 714.0, 714.1, 714.2, and 714.81) between July 1, 2008, and June 30, 2014. Patients were excluded if they were not continuously enrolled in a health plan for a 12-month period after their first RA claim or if they had missing information on their location of residence.

\section{Primary Endpoints: Quality of Care}

Two RA-related measures of health care quality were selected that have been endorsed and validated by national quality measurement stewards and medical organizations: use of DMARDs and TB screening before the initiation of a biologic DMARD ${ }^{7,15,16}$ Nonbiologic DMARDs included methotrexate, as well as auranofin, azathioprine, cyclophosphamide, cyclosporine, hydroxychloroquine, leflunomide, minocycline, mycophenolate, penicillamine, and sulfasalazine. Biologic DMARDs included abatacept, adalimumab, anakinra, certolizumab, etanercept, golimumab, infliximab, rituximab, and tocilizumab. While there is no cure for RA, early diagnosis and appropriate treatment, including the use of DMARDs, can significantly reduce the disease burden and improve patient outcomes. ${ }^{17}$ The DMARD use measure, calculated as the percentage of patients who were diagnosed with RA and prescribed a DMARD, is a Healthcare Effectiveness Data and Information Set (HEDIS) measure used by commercial health plans and also by the Centers for Medicare \& Medicaid Services (CMS) in their Medicare Advantage star ratings system. ${ }^{18}$ For the DMARD measure, patients were required to be continuously enrolled for a 12-month period between July 1 and June 30 of the following year. We then categorized metropolitan statistical areas (MSAs) into 5 groups based on the share of patients with RA who used a DMARD during the year. These groups corresponded to the 2016 Medicare Advantage star rating thresholds, ${ }^{18}$ where group 1 corresponded to DMARD use by $<64 \%$ of RA patients; group 2 corresponded to $\geq 64 \%$ but $<72 \%$ DMARD use; group 3 corresponded to $\geq 72 \%$ but $<75 \%$ DMARD use; group 4 corresponded to $\geq 75 \%$ but $<86 \%$ DMARD use; and group 5 corresponded to $\geq 86 \%$ DMARD use.

For patients who do not respond to DMARDs, biologic DMARDs are recommended as a next-line therapy. ${ }^{19}$ Patients who are administered biologic DMARDs, however, are at a higher risk of TB reactivation, which physicians can mitigate by screening for TB before prescribing a biologic DMARD. ${ }^{20}$ As a result, medical organizations and policymakers have endorsed and used the TB test metric, which measures the percentage of patients who have documentation of a TB test 6 months before initiating a biologic DMARD. ${ }^{15,21}$ This measure is part of CMS's Physician Quality Reporting System, which promotes reporting of quality information by health care professionals. ${ }^{22}$ For the TB test quality metric, patients were required to be continuously enrolled 6 months before and 12 months after their first biologic DMARD prescription observed in the data (i.e., a 6-month window was required with no biologic DMARD use).

\section{Secondary Endpoints: Access to Care}

To understand how well different regions diagnose RA and transition patients to appropriate care, we also calculated RA measured detection and the share of patients who received care from a rheumatologist. The measured detection metric was calculated as the percentage of patients aged $\geq 18$ years diagnosed with RA during the measurement period. In our data, measured detection and prevalence are indistinguishable. If the true demographics-adjusted prevalence of RA is similar across MSAs, then any differences in RA detection in the data likely reflect differences in patient access to providers that can 
correctly diagnose RA. The latter metric was calculated as the percentage of patients diagnosed with RA who received treatment by a rheumatologist. RA patients were required to have $\geq 1$ rheumatologist visit on or after the diagnosis visit during the measurement period. We measured RA patient access to rheumatologists, since recent studies indicate that patients treated by specialists had improved health outcomes compared with patients who were not. ${ }^{23}$

\section{Tertiary Endpoints: Cost of Care and Resource Utilization}

To determine whether patient quality of care was related to the cost of treating these patients, their annual cost of care was also measured. The annual cost measure (inflated to 2015 U.S. dollars using the Consumer Price Index) included payments from insurers as well as all beneficiary out-of-pocket payments. ${ }^{24}$ Costs were further divided into inpatient, outpatient, and pharmacy costs, and medical (i.e., nonpharmacy) costs were categorized as RA related and non-RA related, based on whether the medical claims included diagnosis codes for RA. Finally, the average annual number of hospitalizations (all cause and RA related) and emergency room (ER) visits were computed to measure acute care service use.

\section{Geographic Definitions and Other Variables}

A 2-step process was used to measure quality by different geographic areas. First, we constructed each quality and utilization metric at the individual level, and then we aggregated the quality measures by geographic area defined as an MSA. Developed by the Office of Management and Budget, MSAs represent geographic regions with high population densities and close economic integration. MSAs are comprised of individual counties and equivalent entities. If a county was not included within an existing MSA, we assigned it to a residual "rest of state" area consisting of all non-MSA counties in a state. CMS uses this MSA/rest-of-state definition to define geographic regions as part of their hospital wage index. ${ }^{25}$ Because MSAs are defined as combinations of counties, we aggregated the county-level socioeconomic measures from the AHRF to MSAs, weighting the measures based on the total population in each county within its MSA.

\section{Statistical Analysis}

The objective of this analysis was to characterize geographic variation in quality of care, access to care, and health care spending. First, geographic variations in the DMARD and TB quality metrics were evaluated using logistic regression, adjusting for a patient's age and gender. Second, relationships between geographic variation and resource use (i.e., hospitalization and ER visits) were assessed using negative binomial regression controlling for differences in patient age, gender, and health status (i.e., Elixhauser Comorbidity Inde $\mathrm{x}^{26}$ ). Third, the cost and utilization outcomes, such as inpatient costs or RA total costs, were estimated using linear regression adjusting for a patient's age and gender. All regression models included a year of enrollment indicator variable to control for secular trends.

For the DMARD use and TB quality measures, we estimated the predicted MSA-specific values based on the sample's national average age and gender. To benchmark the DMARD use measure, we classified MSAs into 5 groups that corresponded to Medicare Advantage's 2016 "RA Management" star rating scale. ${ }^{18}$ For the access measure describing the share of patients who received care from a rheumatologist, all regressions accounted for differences across regions in the age/gender composition of patients with RA. For the measured detection access metric, we accounted for differences in the age/gender composition of all patients in an MSA rather than just those with RA.

The relationship between the MSA-level risk-adjusted RA quality measures and MSA-level risk-adjusted health economic outcomes was assessed using Pearson's product-moment correlation. To ensure that the analysis was nationally representative, all analyses were weighted by the age/gender composition with each MSA, as reported in the 2013 Current Population Survey. Variability across MSAs in the quality of care, access to care, and cost of care and resource utilization measures was measured using interquartile range (IQR) and the coefficient of variation $(\mathrm{CV})$.

\section{Results}

After applying the inclusion restrictions separately for the patient-year cohort, the study sample included 364,371 RA patient-years in the commercial claims data and 137,005 from the Medicare data-501,376 in total (Figure 1). Patients who were continuously enrolled for multiple 12-month periods were included in the sample multiple times. For patients initiating biologic DMARD therapy who would be eligible for the TB screening quality measure, 28,642 unique patients met the inclusion criteria in the commercial data, and 5,556 met the inclusion criteria in the Medicare data-a total of 34,198 unique patients. We assigned these patients to 1 of $430 \mathrm{MSAs}$, including 383 actual MSAs and 47 "rest of state" MSAs.

Among prevalent RA patients, the mean age was 57.3 years, and $75.7 \%$ were female (Table 1). The most common comorbidities were uncomplicated hypertension (45.4\%), chronic pulmonary disease (18.8\%), hypothyroidism (18.6\%), diabetes (16.3\%), and depression (14.3\%). For the RA patients, $64.1 \%$ used a DMARD in the previous year, and $29.6 \%$ used a biologic DMARD. About half (57.6\%) of the patients had visited a rheumatologist during the previous year.

Patients who initiated a biologic DMARD therapy were slightly younger (53.1 years), but there was a similar share of female patients in the prevalent cases. The occurrence of common comorbidities was similar for the biologic DMARD new start cohort and the prevalent RA cohort. Just under one third (31.0\%) of patients initiating a biologic DMARD therapy received a TB test. 


\section{FIGURE 1 Study Cohort}

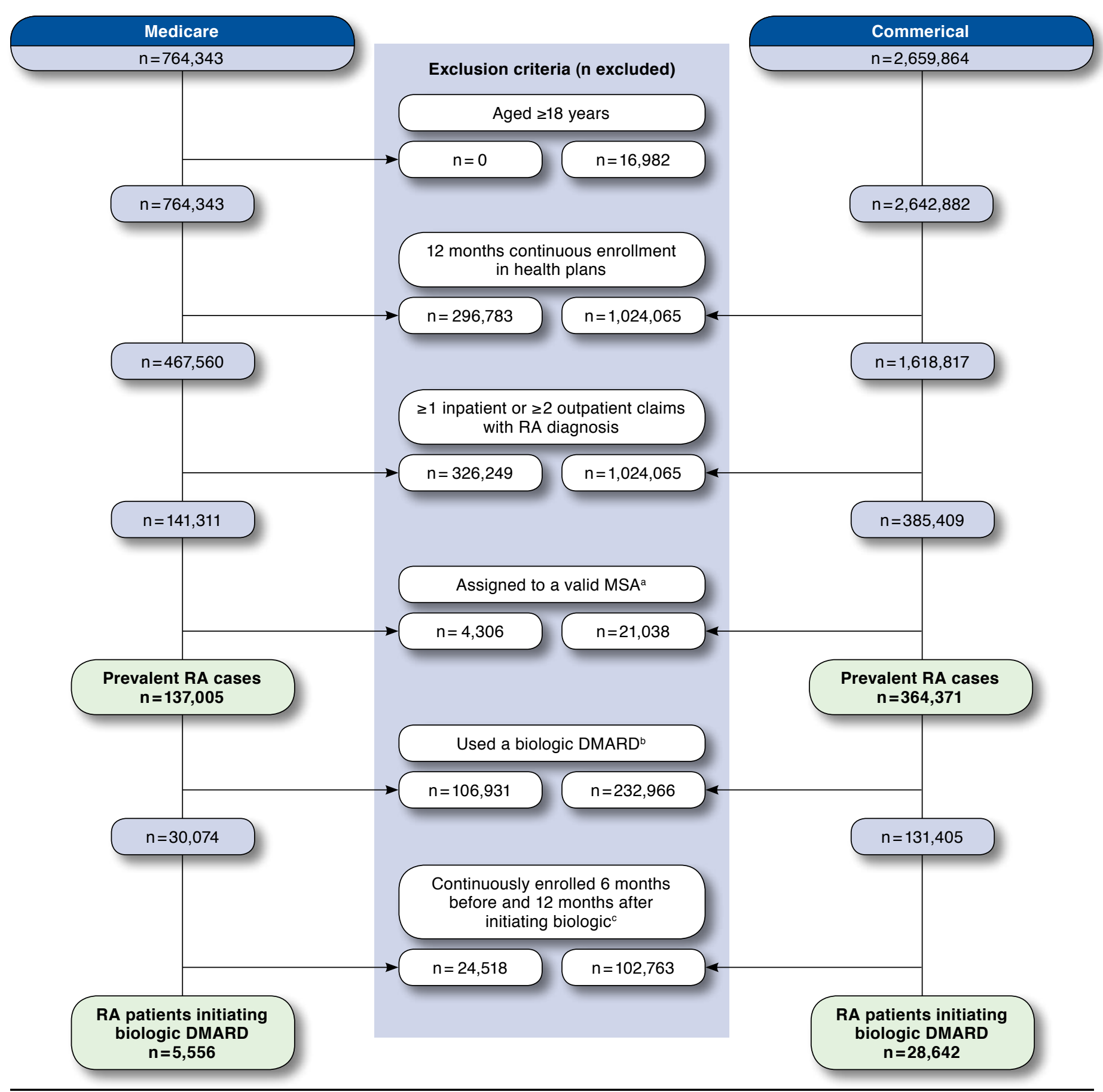

a Patients were required to have a valid MSA weight from the Current Population Survey.

${ }^{b}$ Patients were required to be aged $\geq 18$ years at initiation of therapy.

cPatients were also required to have $\geq 1$ inpatient or $\geq 2$ outpatient claims with an RA diagnosis at initiation of therapy and valid MSA with valid MSA weight from the Current Population Survey.

$D M A R D=$ disease-modifying antirheumatic drug; $M S A=$ metropolitan statistical area; $R A=$ rheumatoid arthritis. 


\section{TABLE 1}

Patient Characteristics for 2 Quality Measure Cohorts

\begin{tabular}{|c|c|c|}
\hline Category & $\begin{array}{c}\text { Eligible for } \\
\text { DMARD Use } \\
\text { Quality Measure } \\
\% \text { (n) or mean }[S D]\end{array}$ & $\begin{array}{c}\text { Eligible for TB } \\
\text { Test Quality } \\
\text { Measureb } \\
\% \text { (n) or mean [SD] }\end{array}$ \\
\hline \multicolumn{3}{|l|}{ Demographics } \\
\hline Female & $75.7(379,392)$ & $76.7 \quad(26,498)$ \\
\hline Age & $57.3 \quad[13.8]$ & $53.1 \quad[12.7]$ \\
\hline \multicolumn{3}{|l|}{ Quality measures } \\
\hline DMARD use (all DMARDs) & $64.1(321,435)$ & $100.0(34,535)$ \\
\hline DMARD use (biologic DMARDs) & $29.6(148,372)$ & $100.0(34,535)$ \\
\hline TB test & & $31.0 \quad(10,695)$ \\
\hline \multicolumn{3}{|l|}{ Access measures } \\
\hline RA prevalence & $0.6(501,376)$ & \\
\hline Rheumatologist visit & $57.6(288,590)$ & $69.2 \quad(23,915)$ \\
\hline \multicolumn{3}{|l|}{ Cost } \\
\hline Inpatient & $5,257 \quad[25,157]$ & $4,750 \quad[28,197]$ \\
\hline Outpatient & $11,394 \quad[22,215]$ & $17,636 \quad[24,009]$ \\
\hline Pharmacy & $5,849 \quad[10,796]$ & $17,782 \quad[15,600]$ \\
\hline Total & $22,501 \quad[38,419]$ & $40,168 \quad[39,523]$ \\
\hline \multicolumn{3}{|l|}{ RA-related cost } \\
\hline Inpatient & $2,326 \quad[12,285]$ & $2,103 \quad[14,057]$ \\
\hline Outpatient & $3,683 \quad[12,031]$ & $9,412 \quad[16,380]$ \\
\hline Total & $6,009 \quad[17,378]$ & $11,515 \quad[21,846]$ \\
\hline \multicolumn{3}{|l|}{ Elixhauser comorbidities ${ }^{c}$} \\
\hline Hypertension, uncomplicated & $45.4(227,811)$ & $44.2 \quad(15,254)$ \\
\hline Chronic pulmonary disease & $18.8(94,375)$ & $20.0 \quad(6,920)$ \\
\hline Hypothyroidism & $18.6 \quad(93,243)$ & $(6,835)$ \\
\hline Diabetes, uncomplicated & $16.3(81,639)$ & $(5,533)$ \\
\hline Depression & $14.3(71,609)$ & $(5,885)$ \\
\hline Cardiac arrhythmia & $11.1 \quad(55,758)$ & $(3,597)$ \\
\hline Fluid and electrolyte disorders & $8.2(41,105)$ & $(3,124)$ \\
\hline Obesity & $8.0 \quad(40,251)$ & $(3,459)$ \\
\hline Valvular disease & $7.5 \quad(37,407)$ & $(2,460)$ \\
\hline Deficiency anemia & $7.1 \quad(35,663)$ & $(2,757)$ \\
\hline Peripheral vascular disorders & $7.0(34,864)$ & $(2,037)$ \\
\hline Solid tumor without metastasis & $6.2(31,195)$ & $(1,711)$ \\
\hline Heart failure & $5.3(26,771)$ & $(1,426)$ \\
\hline Liver disease & $5.1 \quad(25,393)$ & $7.3 \quad(2,519)$ \\
\hline Total, $\mathrm{n}$ & $501,376^{\mathrm{d}}$ & 34,198 \\
\hline \multicolumn{2}{|c|}{ ancludes patients with prevalent cases of $R A$. } & ${ }^{b}$ Includes patients with RA that initiated a biologic DMARD. \\
\hline \multicolumn{3}{|c|}{$\begin{array}{l}\text { 'Greater than 5\% of patients with RA had the following Elixhauser comorbidities: } \\
\text { alcohol abuse, blood loss anemia, coagulopathy, diabetes complicated, drug abuse, } \\
\text { HIV, hypertension, complicated, lymphoma, metastatic cancer, other neurological } \\
\text { disorders, paralysis, peptic ulcer disease, psychoses, pulmonary circulation disor- } \\
\text { ders, renal failure, and weight loss. } \\
\text { dData file contained } 501,376 \text { patients with RA. From the complete data file, we } \\
\text { also received the total number of individuals by MSA, by gender, and by 10-year } \\
\text { age grouping. These counts were used to calculate the total prevalence. } \\
\text { DMARD = disease-modifying antirheumatic drug; MSA=metropolitan statistical } \\
\text { area; RA= rheumatoid arthritis; SD= standard deviation; TB= tuberculosis. }\end{array}$} \\
\hline
\end{tabular}

\section{Quality of Care Results}

In the average MSA, $64.5 \%$ (IQR $=58.7 \%-72.2 \%, C V=0.161)$ of patients received a DMARD, while 28.9\% (IQR $=20.6 \%-36.0 \%$, $\mathrm{CV}=0.450$ ) of patients who were prescribed a biologic DMARD received a TB test within 6 months before treatment initiation. Further, MSAs at the 90th quality percentile had DMARD prescribing rates of $77.3 \%$ for patients with RA, compared with $50.7 \%$ for MSAs at the 10th quality percentile. States at the 90th quality percentile conducted TB tests for $45.5 \%$ of patients initiating a biologic DMARD compared with 14.3\% of states at the 10th percentile. These results suggest that a large share of patients with RA receive a standard of care below accepted guidelines, even in the highest quality MSAs.

When the DMARD-use quality metric was divided into 5 groups based on the Medicare Advantage quality star rating system, the majority of MSAs had low levels of DMARD use relative to established benchmarks. Seventeen percent (73/430) of MSAs had the highest share of RA patients using DMARDs (i.e., groups 4 and 5), which would correspond to the region receiving 4 or 5 stars if the MSA was ranked using the Medicare Advantage star ratings scale. ${ }^{18}$ By comparison, $74.4 \%$ (357/430) of MSAs had suboptimal DMARD use, as indicated by belonging to the 3 lowest quality groups (groups 1, 2, or 3). On average, metropolitan areas (64.7\%) performed better than nonmetropolitan areas (62.9\%).

MSAs with higher quality care (based on DMARD use) had significantly lower poverty rates, higher education, and fewer Medicaid-eligible residents. Average family income and the share of minority residents in an MSA, however, were not associated with an MSA's quality of care.

Regions that ranked highly on the DMARD use measure, however, were not necessarily those that ranked highly on the TB test quality measure. There was near zero correlation (Pearson correlation:-0.02, P=0.125) found between an MSA's DMARD use and its likelihood of conducting TB tests before initiating biologic therapy. For instance, Hot Springs, Arkansas, was the top MSA in terms of DMARD use but was second to worst at TB testing among patients initiating a biologic therapy. Sheboygan, Wisconsin, had the second-highest DMARD-prescribing rate but ranked in the middle for TB testing (175/430). New York City, which ranked towards the bottom in DMARD prescriptions (410/430), actually was in the top quintile in terms of TB prescribing (59/430).

\section{Access to Care Results}

Similar levels of variability were found based on access to care measures. Measured detection of RA in claims data was 0.59\% (IQR $=0.47 \%-0.71 \%, C V=0.355)$, and the share of patients with RA who visited a rheumatologist during the year was only $57.6 \%(\mathrm{IQR}=47.9 \%-69.4 \%, \mathrm{CV}=0.286)$.

Most regions had both low quality of care and access to care. A visual comparison between DMARD use and measured 


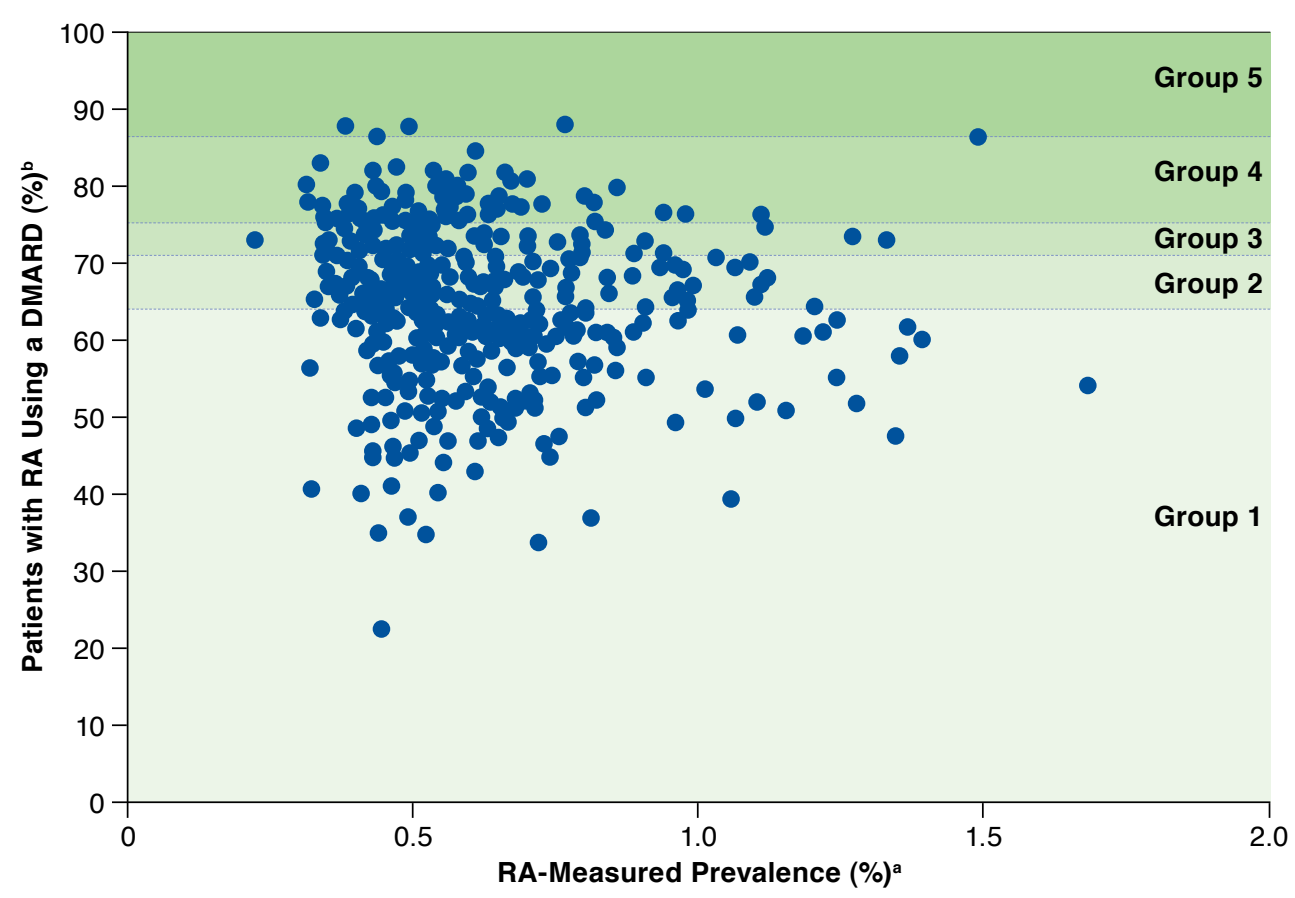

ancludes all data points with $\geq 200$ patients with $R A(n=409)$.

${ }^{b}$ The DMARD use groupings correspond to the 2016 Medicare Advantage (MA) star rating system that assigns MA plans a 1-5 star rating based on the share of patients who receive a DMARD. Group 5 corresponds to a 5-star rating, and Group 1 corresponds to a 1-star rating.

DMARD = disease-modifying antirheumatic drug; $R A=$ rheumatoid arthritis .

detection indicates that there are many areas with low quality of care, and even among the higher quality regions, many areas appear to have poor access to care (Figure 2). Further, the correlation between a region's access to care and quality of care was fairly weak. For instance, the correlation between measured detection and DMARD use across regions was -0.10, and the correlation between the share of patients visiting a rheumatologist and DMARD use was -0.03 .

\section{Relationship Between Quality and Cost}

MSAs that provided high-quality RA care had higher total spending for RA patients but lower RA-related inpatient expenditures (Table 2). MSAs with the highest DMARD use spent $\$ 26,724$, on average, treating patients with RA, whereas lowquality MSAs spent only $\$ 21,296$ ( $\$ 5,428$ difference, $P<0.001$ ). Overall inpatient costs for RA patients were $\$ 629$ higher in MSAs with the most DMARD use than in the low-quality MSAs (group 5: $\$ 5,885$ vs. group 1: $\$ 5,255, P=0.412$ ). The cost of RA-related inpatient care was $\$ 554$ less in high quality MSAs than in the lowest quality MSAs (group 5: $\$ 1,890$ vs. group 1: $\$ 2,417, P=0.024$ ).

\section{Discussion}

We compared geographic variations across the United States in the quality of care, access to care, and total spending for patients with RA. We observed that the quality of care as currently practiced is suboptimal across most of the United States according to CMS measures of RA quality of care. ${ }^{7}$ Many patients with RA are not receiving DMARDs, and among those who receive biologic DMARD treatments, fewer than half receive a recommended TB test. This conclusion echoes previous work, which concluded that although DMARDs are a widely accepted treatment for RA, they are significantly underused in practice. ${ }^{27}$

We also observed significant variation in quality and access to care across MSAs. In MSAs at the 90th percentile of the quality distribution, almost 4 in 5 RA patients received a DMARD, whereas at the 10th percentile quality level, the figure was about 1 in 2 . Similar to the findings from a previous Institute of Medicine report, ${ }^{28}$ we found significant variation in quality within broadly defined regions (Figure 3). Some MSAs were significantly better than others in terms of ensuring that RA patients initiating biologic DMARD therapy received a TB test. Access to care metrics-measured detection and share of 
Geographic Variation in the Quality and Cost of Care for Patients with Rheumatoid Arthritis

TABLE 2 MSA Annual Cost Per RA Patient by Star Rating Group on DMARD Use Quality Measure

\begin{tabular}{|c|c|c|c|c|c|}
\hline Cost/Resource Use [SD] & $\begin{array}{l}\text { Group 1 } \\
(\mathrm{n}=198)\end{array}$ & $\begin{array}{l}\text { Group } 2 \\
(n=121)\end{array}$ & $\begin{array}{c}\text { Group } 3 \\
(\mathrm{n}=38)\end{array}$ & $\begin{array}{c}\text { Group } 4 \\
(n=68)\end{array}$ & $\begin{array}{c}\text { Group } 5 \\
(\mathrm{n}=5)\end{array}$ \\
\hline \multicolumn{6}{|l|}{ Overall cost } \\
\hline Inpatient & $5,255 \quad[2,151]$ & $5,307 \quad[1,958]$ & $5,409 \quad[1,654]$ & $5,206 \quad[1.879]$ & $5,885 \quad[2,603]$ \\
\hline (vs. Group 1)a & - & 52 & 154 & (49) & 629 \\
\hline Outpatient & $10,951 \quad[2,813]$ & $11,617 \quad[2,360]$ & $12,331 \quad[3,048]$ & $12,543 \quad[4,351]$ & $13,229[4,085]$ \\
\hline$($ vs. Group 1)a & - & 666 & 1,380 & 1,592 & 2,279 \\
\hline Pharmacy & $5,090 \quad[1,542]$ & $6,156 \quad[1,078]$ & $6,444 \quad[1,434]$ & $6,774 \quad[1,827]$ & $7,610 \quad[1,511]$ \\
\hline$($ vs. Group 1)a & - & 1,066 & 1,353 & 1,683 & 2,520 \\
\hline Total & $21,296 \quad[4,601]$ & $23,080 \quad[3,713]$ & $24,183 \quad[4,328]$ & $24,523 \quad[5,146]$ & $26,724 \quad[5,438]$ \\
\hline (vs. Group 1)a & - & 1,784 & 2,887 & 3,227 & 5,428 \\
\hline \multicolumn{6}{|l|}{ RA-related $\cos t^{b}$} \\
\hline Inpatient & $2,417 \quad[1,332]$ & $2,342 \quad[984]$ & $2,241 \quad[945]$ & $2,211 \quad[847]$ & $1,890 \quad[653]$ \\
\hline$($ vs. Group 1)a & - & $(75)$ & $(175)$ & (206) & $(527)$ \\
\hline Outpatient & $3,360 \quad[1,537]$ & $3,722 \quad[1,392]$ & $3,711 \quad[1,530]$ & $4,173 \quad[2,451]$ & $4,666 \quad[2,279]$ \\
\hline$($ vs. Group 1)a & - & 362 & 350 & 812 & 1,306 \\
\hline \multicolumn{6}{|l|}{ Utilization } \\
\hline Hospitalizations per year & $0.15 \quad[0.035]$ & $0.15 \quad[0.027]$ & $0.15 \quad[0.027]$ & $0.16 \quad[0.037]$ & $0.17 \quad[0.062]$ \\
\hline (vs. Group 1)a & - & - & - & 0.01 & 0.02 \\
\hline RA-related hospitalizations per year & $0.08 \quad[0.023]$ & $0.08[0.020]$ & $0.07 \quad[0.021]$ & $0.08 \quad[0.029]$ & $0.06 \quad[0.032]$ \\
\hline (vs. Group 1)a & - & - & $(0.01)$ & - & $(0.02)$ \\
\hline ER visits per year & $0.33[0.087]$ & $0.32[0.078]$ & $0.29[0.072]$ & $0.30 \quad[0.093]$ & $0.31 \quad[0.142]$ \\
\hline$($ vs. Group 1)a & - & $(0.01)$ & $(0.04)$ & $(0.03)$ & $(0.02)$ \\
\hline \multicolumn{6}{|c|}{$\begin{array}{l}\text { Source: This table shows analysis results using data on patients with RA from Truven MarketScan Medicare and Commercial claims data, July } 2008 \text {-June } 2013 \text {. All figl } \\
\text { were inflated to } 2015 \text { USD using the Consumer Price Index. } \\
\text { aThe groups correspond to the } 2016 \text { Medicare Advantage star ratings, where group } 1 \text { is DMARD use }<64 \% \text { among RA patients; group } 2 \text { is } \geq 64 \% \text { but }<72 \% \text {; group } 3 \text { is } \\
\geq 72 \% \text { but }<75 \% \text {; group } 4 \text { is } \geq 75 \% \text { but }<86 \% \text {; and group } 5 \text { is } \geq 86 \% \text {. } \\
\text { bRA-related cost is defined as the sum of the cost on medical claims that have an RA diagnosis code. } \\
\text { DMARD = disease-modifying antirheumatic drug; ER=emergency room; RA=rheumatoid arthritis; SD=standard deviation; USD=U.S. dollars. }\end{array}$} \\
\hline
\end{tabular}

patients visiting a rheumatologist—also varied significantly across regions, but the correlation between access to care and quality of care was fairly weak.

Note that our analysis did not tell us why DMARD use and TB screening were suboptimal. For example, DMARD use could be low because too few RA patients are being prescribed a DMARD by their physicians, or it could be that demand for DMARDs is low because of cost sharing or some other explanation, such as side-effect risk. Although there are many evidence-based guidelines, additional research is needed to better understand why these practices have not been adopted.

When comparing the association between measured quality of RA treatment and the cost of care, we found that there was a trade-off between higher quality and higher cost. MSAs with a higher proportion of patients receiving DMARDs spent more on care overall, largely because of additional outpatient and prescription drug costs, but had lower RA-related inpatient expenditures.

It is important to note that we only estimated an association between quality of care and patient outcomes and spending. It is possible that the negative association between high DMARD use and a lower RA-related hospitalization rate was entirely driven by unobserved differences in the patient population. In particular, our results may not fully explain what would happen to a given MSA's RA hospitalization rate if it increased the percentage of patients receiving DMARDs. The correlation between MSA health care expenditures and DMARD use may be caused by a provider culture that takes a more interventionist approach; DMARD use itself may decrease inpatient cost, since we see that RA-related inpatient costs were lower in the regions with the highest DMARD use. However, from the standpoint of using the quality measure as a tool to sort through a group of MSAs, our findings suggest that MSAs with higher quality of care (distinguished as MSAs with more DMARD use and better TB screening) are more likely to have, on average, higher costs but also better patient outcomes.

\section{Limitations}

This study has a number of limitations. First, the MarketScan data are a convenience sample. To extrapolate the regression results from the MarketScan sample to the broader U.S. population, we followed past work studying geographic variations using private claims data, ${ }^{29}$ calculated age-gender stratum weights using national survey data, and applied them to 


\section{A. Proportion of patients with RA who received a DMARD} during the year, 2008-2014

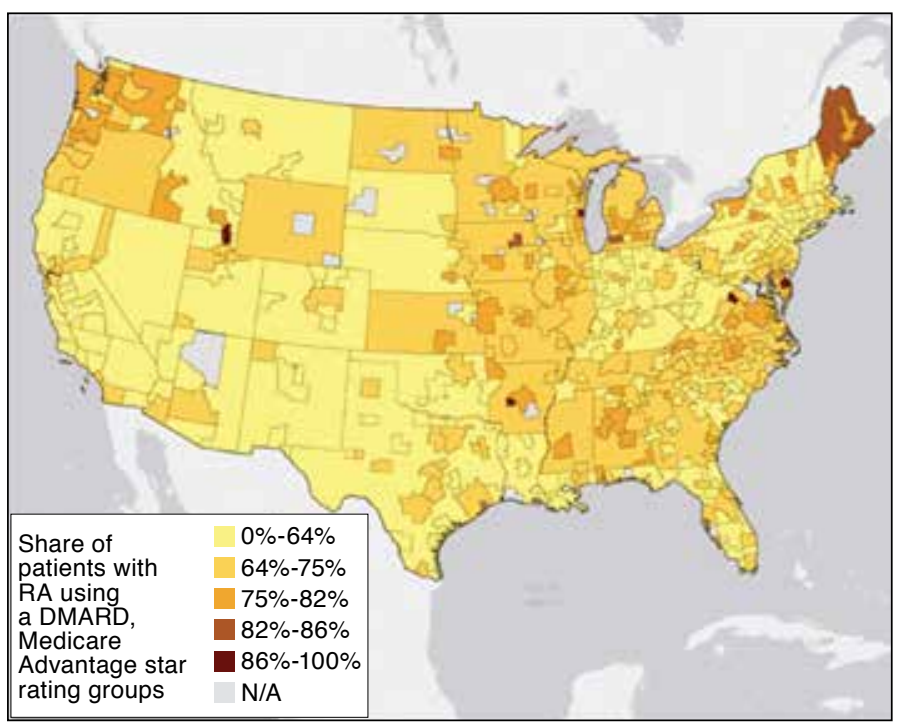

\section{B. TB screening among RA patients who initiated a biologic} DMARD, 2008-2014

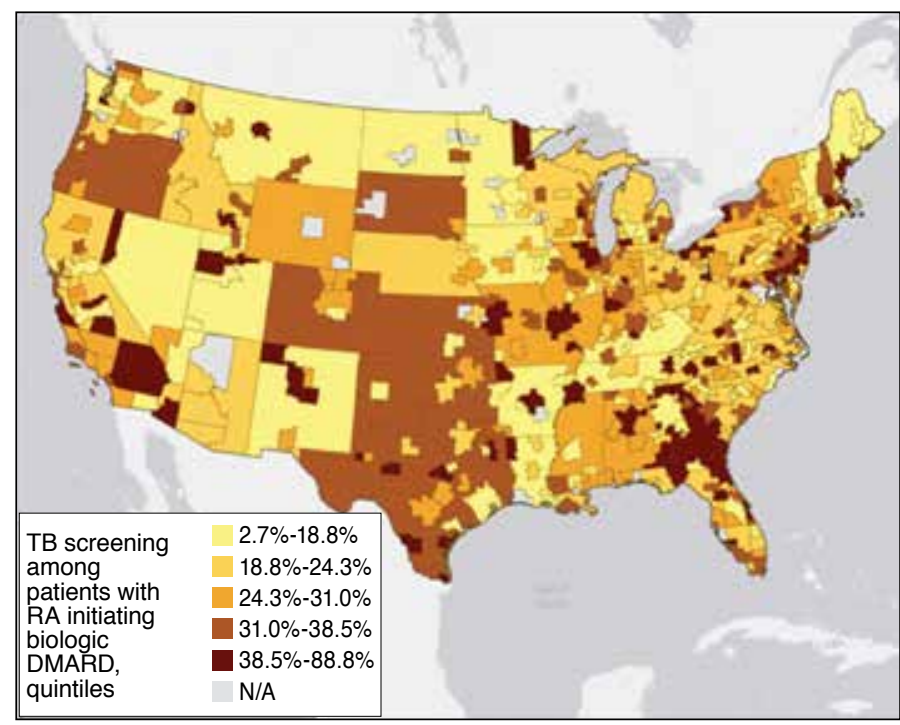

Source: Analysis using data on patients with RA from Truven MarketScan Medicare and Commercial claims data, July 2008-June 2014. Star ratings thresholds are from CMS documentation.

aPanel A shows the geographic variation in DMARD use, and Panel B shows the geographic variation in TB screening before initiating a biologic DMARD. $C M S=$ Centers for Medicaid \& Medicare Services; DMARD=disease-modifying antirheumatic drug; N/A=not available; $R A=$ rheumatoid arthritis; $T B=$ tuberculosis

regression models. Specifically, we constructed weights by dividing the population estimates from the Current Population Survey in 2013 by the number of beneficiaries in the MarketScan study sample for each age-gender stratum in a given MSA (using age groups 0-9 years, 10-19 years, 20-29 years, 30-44 years, 45-54 years, 55-64 years, 65-74 years, and $\geq 75$ years for males and females). Essentially, this approach put more or less weight on observations in age-gender bins that were less or more common in the data relative to the general population.

Second, rather than using MSAs as the basis for analysis, many studies_-such as the Dartmouth Atlas suite of projectsrely on hospital referral regions (HRR) to define geographic regions rather than MSAs. Defining HRRs requires ZIP code information, which was not reported in the MarketScan data. However, research examining geographic variation in health care spending found that geographic variation in Medicare spending was of similar magnitude whether measured using HRRs or MSAs. ${ }^{29}$

Third, another limitation common to all claims-based analysis is that important clinical data needed to measure RA severity was not available. Although we cannot rule out that some of the observed geographic variation in health care quality, spending, or outcomes was because of unobserved differences in RA disease severity, many of the predictors of
RA-related infections-including diabetes mellitus, alcohol abuse, and chronic pulmonary disease-are already included within the list of Elixhauser comorbidities used. ${ }^{30}$

Fourth, we were not able to separate whether observed differences in RA detection across MSAs are because of variation in patient access to care or variation in baseline RA prevalence across MSAs. Finally, this study measured the correlation between quality and cost, and the results should not be interpreted as estimates of the causal relationship between quality measures and cost or outcomes.

\section{Conclusions}

We found that quality of care in patients with RA is not only fairly low on average, but also varies significantly across the United States. While many new health care reimbursement mechanisms, such as accountable care organizations, are based on the premise that providers can produce higher quality of care at lower costs, our study indicates this may not be an achievable goal across all diseases. As a chronic disease, higher RA-related prescription and outpatient costs may be only partially offset by reduced inpatient costs, but there are likely unmeasured benefits from reduced losses of productivity and improved patient outcomes and quality of life. Efforts to increase value in health care need to balance efforts to contain 
costs with the reality that, in some cases, providing higher quality of care and improving patient outcomes requires higher spending.

\section{Authors}

JASON SHAFRIN, PhD, and JIN JOO SHIM, MS, Precision Health Economics, Los Angeles, California. ARIJIT GANGULI, MBA, PhD, and YURI SANCHEZ GONZALEZ, PhD, AbbVie, North Chicago, Illinois. SETH A. SEABURY, PhD, Keck School of Medicine, University of Southern California, Los Angeles.

AUTHOR CORRESPONDENCE: Jason Shafrin, PhD, Precision Health Economics, 11100 Santa Monica Blvd., Los Angeles, CA 90025. Tel.: 310.984.7705; E-mail: jason.shafrin@pheconomics.com.
6. Centers for Medicare \& Medicaid Services. Medicare 2017 Part C $\&$ D star rating technical notes: first plan preview. August 3, 2016. Available at: https://www.cms.gov/Medicare/Prescription-Drug-Coverage/ PrescriptionDrugCovGenIn/Downloads/2017_Technical_Notes_preview_1_2016_08_03.pdf. Accessed October 28, 2016.

7. Centers for Medicare \& Medicaid Services. Physician Quality Reporting System. 2014. Available at: http://www.cms.gov/Medicare/QualityInitiatives-Patient-Assessment-Instruments/pqrs/index.html. Accessed October 15, 2016

8. DeMaria L, Acelajado MC, Luck J, et al. Variations and practice in the care of patients with rheumatoid arthritis: quality and cost of care. J Clin Rheumatol. 2014;20(2):79-86.

9. Harrold LR, Harrington JT, Curtis JR, et al. Prescribing practices in a US cohort of rheumatoid arthritis patients before and after publication of the American College of Rheumatology treatment recommendations. Arthritis Rheum. 2012;64(3):630-38.

10. Gawande A. The cost conundrum. The New Yorker. June 1, 2009. Available at: http://www.newyorker.com/magazine/2009/06/01/the-costconundrum. Accessed October 15, 2016.

11. Birkmeyer JD, Gust C, Dimick JB, Birkmeyer NJ, Skinner JS. Hospital quality and the cost of inpatient surgery in the United States. Ann Surg. 2012;255(1):1-5.

12. Baicker K, Chandra A. Medicare spending, the physician workforce and beneficiaries quality of care. Health Aff (Millwood). Published online April 7, 2004. Available at: http://content.healthaffairs.org/content/early/2004/04/07/ hlthaff.w4.184.long. Accessed October 15, 2016.

13. Hussey PS, Wertheimer S, Mehrotra A. The association between health care quality and cost: a systematic review. Ann Intern Med. 2013;158(1):27-34

14. U.S. Department of Health \& Human Services, Health Resources and Services Administration. Area Health Resources Files. 2013. Available at: http://ahrf.hrsa.gov/. Accessed October 28, 2016.

15. American College of Rheumatology. ACR endorsed measures. Rheumatoid arthritis. Available at: http://www.rheumatology.org/PracticeQuality/Clinical-Support/Quality-Measurement/ACR-Endorsed-Measures. Accessed October 28, 2016.

16. National Quality Forum. Quality positioning system. 2014. Available at: http://www.qualityforum.org/QPS. Accessed October 15, 2016.

17. Strand V, Singh JA. Improved health-related quality of life with effective disease-modifying antirheumatic drugs: evidence from randomized controlled trials. Am J Manag Care. 2008;14(4):234-54.

18. Centers for Medicare \& Medicaid Services. Part C and D Performance Data. 2016 Part C and D Medicare Star Ratings Data (v4 07 2016). 2015. Available at: https://www.cms.gov/medicare/prescription-drug-coverage/prescriptiondrugcovgenin/performancedata.html. Accessed October 15, 2016.

19. Singh JA, Furst DE, Bharat A, et al. 2012 Update of the 2008 American College of Rheumatology recommendations for the use of disease-modifying antirheumatic drugs and biologic agents in the treatment of rheumatoid arthritis. Arthritis Care Res (Hoboken). 2012;64(5):625-39.

20. Scrivo R, Armignacco O. Tuberculosis risk and anti-tumour necrosis factor agents in rheumatoid arthritis: a critical appraisal of national registry data. Int J Rheum Dis. 2014;17(7):716-24.

21. American Medical Association. Physician consortium for performance improvement. 2014. Available at: http://www.ama-assn.org/ama/pub/ physician-resources/physician-consortium-performance-improvement/pcpimeasures.page. Accessed October 15, 2016.

22. Centers for Medicare \& Medicaid Services. 2014 Physician Quality Reporting System (PQRS) measures list. December 15, 2013. Available at: https://www.facs.org/ /media/files/advocacy/pqrs/2014/pqrs\%20measures\%20list.ashx. Accessed October 15, 2016. 
23. Yelin EH, Such CL, Criswell LA, Epstein WV. Outcomes for persons with rheumatoid arthritis with a rheumatologist versus a non-rheumatologist as the main physician for this condition. Med Care. 1998;36(4):513-22.

24. U.S. Bureau of Labor Statistics. Consumer Price Index: CPI databases. 2014. Available at: http://www.bls.gov/cpi/data.htm. Accessed October 15, 2016.

25. MaCurdy T, Shafrin J, DeLeire T, et al. Geographic adjustment of Medicare payments to physicians: evaluation of IOM recommendations. Acumen LLC. July 2012. Available at: https://www.cms.gov/medicare/ medicare-fee-for-service-payment/physicianfeesched/downloads/geographic_adjustment_of_medicare_physician_payments_july2012.pdf. Accessed October 15, 2016.

26. Quan H, Sundararajan V, Halfon P, et al. Coding algorithms for defining comorbidities in ICD-9-CM and ICD-10 administrative data. Med Care. 2005:43(11):1130-39.
27. Solomon DH, Yelin E, Katz JN, Lu B, Shaykevich T, Ayanian JZ. Treatment of rheumatoid arthritis in the Medicare Current Beneficiary Survey. Arthritis Res Ther. 2013;15(2):R43.

28. Institute of Medicine. Crossing the Quality Chasm: A New Health System for the 21st Century. Washington, DC: The National Academies Press; 2001.

29. Philipson TJ, Seabury SA, Lockwood LM, Goldman DP, Lakdawalla DN. Geographic variation in health care: the role of private markets. In: Romer DH, Wolfers J, eds. Brookings Papers on Economic Activity: Spring 2010.

Washington, DC: Brookings Institution Press; 2010:325-55. Available at: https://www.brookings.edu/wp-content/uploads/2010/03/2010a_bpea_philipson.pdf. Accessed October 15, 2016.

30. Doran MF, Crowson CS, Pond GR, O'Fallon WM, Gabriel SE. Predictors of infection in rheumatoid arthritis. Arthritis Rheum. 2002;46(9):2294-300. 